\title{
Dramatic change of the recurrence time and outburst parameters of the intermediate polar GK Persei ${ }^{\star}$
}

\author{
V. Šimon \\ Astronomical Institute, Academy of Sciences of the Czech Republic, 25165 Ondřejov, Czech Republic \\ Received 21 May 2001 / Accepted 25 October 2001

\begin{abstract}
This analysis has shown that the intermediate polar GK Per experienced a very striking evolution. Its outbursts became wider and brighter in the last five decades. These changes were accompanied by striking variations of the recurrence time $T_{\mathrm{C}}$, from 385 days within the years 1948-1967 to 890 days in 1970. Nowadays, $T_{\mathrm{C}}$ displays a strong trend of linear increase. Decrease of irradiation of the disk by the WD, combined with the decrease of viscosity, offers a plausible explanation. It is argued that variations of the mass transfer rate are unlikely to play a major role. The morphology of the outburst light curves in the optical and the X-ray region is also studied. The decay branch in the optical remains remarkably similar for all the events while the largest changes of the light curve occur in the rising branch. This can be explained if the thermal instability may start at different distances from the disk center. The quiescent level of brightness does not display any secular trend in recent decades but a wave on the time scale of about 30 years with the full amplitude of $0.3 m_{\mathrm{v}}$, probably due to activity of the cool star, is detected.
\end{abstract}

Key words. stars: activity - binaries: close - binaries: general - stars: magnetic fields - novae, cataclysmic variables - stars: individual: GK Per

\section{Introduction}

GK Per (Nova Per 1901/A 0327+43) is an intermediate polar with $P_{\text {orb }}=1.99$ days (Crampton et al. 1986) and $P_{\text {spin }}=351 \mathrm{~s}$ (Watson et al. 1985). Fluctuations of brightness by about $1 \mathrm{mag}$ on the time scale of months appeared after return to quiescence. Later, they developed into the discrete 2-3 mag optical outbursts which recur on time scales of hundreds of days (e.g. Sabbadin \& Bianchini 1983; Hudec 1981). These optical outbursts are accompanied by brightenings in X-rays (e.g. King et al. 1979). The light curves of three similar outbursts which occurred in 1975, 1981 and 1983 were modeled by Kim et al. (1992) in the framework of the thermal instability disk model. This model was able to reproduce the basic features of the outbursts although some problems remained. Fluctuations of luminosity of 360-5000 s in the different spectral regions were observed during the outbursts (e.g. Duschl et al. 1985; Pezzuto et al. 1996; Morales-Rueda et al. 1996) and attributed mostly to the interaction of the magnetic field

\section{Send offprint requests to: V. Šimon,}

e-mail: simon@asu.cas.cz

* This research has made use of the AFOEV database, operated at CDS, France, and the observations provided by the ASM/RXTE team. of the white dwarf (WD) with the inner disk region or to some sort of low frequency QPO's.

This paper will show the relation between the drastic variations of the energetics of the outbursts and the recurrence times which occurred during the last five decades. Our analysis will also include the last 18 years, not covered by Sabbadin \& Bianchini (1983) and Hudec (1981).

\section{Sources of the data}

Monitoring of dwarf novae is almost entirely the domain of the associations of amateur observers. The observations are mostly visual but they are quite numerous and come from a large number of observers. Visual data, if treated carefully, can be very useful for analysis of long-term activity (Percy et al. 1985; Richman et al. 1994). Accuracy even better than 0.1 mag can be achieved by averaging the data. This is quite sufficient for analyses of these largeamplitude variable stars.

This analysis is partly based on the visual data of GK Per, obtained from the database of AFOEV, operated at CDS, Strasbourg, France. They cover the years 1970-2000. The light curve was plotted and submitted to a visual inspection. Several observations, largely deviating from the neighbouring points on the light curve, were rejected. In order to smooth the light curve, the observations (6005) were then binned into one-day means (2906). 


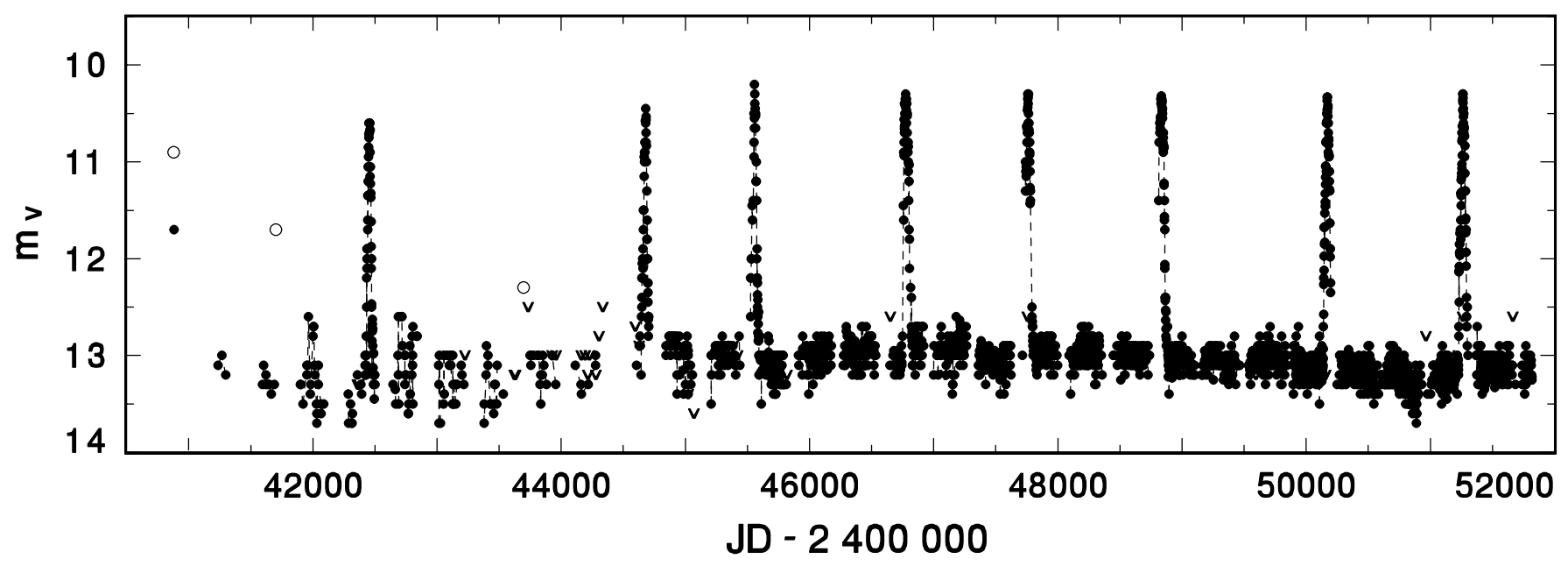

Fig. 1. The outbursts history of GK Per over the years 1970-2000 according to AFOEV observations. Data points are connected by a line for the densely covered intervals. The v symbols represent the upper limits of brightness. Empty circles mark the maxima of three outbursts, which fall in the gaps in the AFOEV data and are listed by Sabbadin \& Bianchini (1983). See Sect. 3.1 for details.

The upper limits of brightness (37) were used to constrain the brightness variations in less densely covered intervals. The result is shown in Fig. 1. All the outbursts after JD 2444500 are unambiguously defined by multiple observations from several nights. The beginning of the interval analyzed here overlaps with the historical light curve by Sabbadin \& Bianchini (1983) till JD 2440000 . The epochs of maxima and the magnitudes of three outbursts, which fall into the gaps in our data but are listed by Sabbadin \& Bianchini (1983), are marked by empty circles in Fig. 1.

The outbursts in 1996 and 1999 were covered by the All Sky Monitor $(A S M)$ onboard the Rossi X-ray Timing Explorer (RXTE) satellite (http://xte.mit.edu/). It was found that GK Per appears faint in the X-ray data (2-12 keV) even at outburst but a careful fitting of the data enabled us to determine at least the main trends (see Sect. 3.3).

\section{Data analysis}

\subsection{General description}

The light curve in Fig. 1 quite resembles that of the dwarf novae of the U Gem-type. The observations are not quite uniformly distributed due to seasonal gaps. We will show that there are good reasons to believe that no outbursts were missed after JD 2444800 . All the outbursts after this date reached about $10.4 \mathrm{~m}_{\mathrm{v}}$, starting from the quiescent value of $\sim 13.2 m_{\mathrm{v}}$, with typical durations of $60-70$ days.

The following analysis of the outbursts is based at a large extent on the methods and procedures applied by Šimon (2000a,b,c) to some dwarf novae.

The moment of the light maximum and the peak magnitude, $m_{\max }$, were determined by fitting a polynomial of the 2nd-4th degree to the upper part of the outburst light curve. Typical errors are $1-2$ days and $0.1 m_{\mathrm{v}}$. Even in the case of incompletely covered light curves the light
Table 1. Parameters of the outbursts in GK Per. $T_{\max }$ refers to the maximum brightness in JD-2 400000 . The epoch number and $\mathrm{O}-\mathrm{C}$ (days) are calculated according to Eqs. (1)-(3); the number of the equation used is listed in the column labelled Eq. The $\mathrm{O}-\mathrm{C}$ variations are so large that in some cases several equations were used for the same outburst to make orientation in Figs. 5a,b,c easier - the respective values are listed in the following line. $T_{\max }, m_{\max }$ and $W$ come from both the measured AFOEV data and from Sabbadin \& Bianchini (1983).

\begin{tabular}{crrrcr}
\hline$T_{\max } \mathrm{JD}$ & Epoch & O-C & Eq. & $m_{\max }$ & $W$ \\
\hline 32758 & -18 & -62 & $(1)$ & 11.0 & 30 \\
33182 & -17 & -23 & $(1)$ & 11.0 & 30 \\
33631 & -16 & 41 & $(1)$ & 11.7 & 40 \\
34782 & -13 & 37 & $(1)$ & 12.0 & 20 \\
35167 & -12 & 37 & $(1)$ & 12.0 & 50 \\
37037 & -7 & -18 & $(1)$ & 11.5 & 40 \\
37405 & -6 & -35 & $(1)$ & 11.5 & 30 \\
39360 & -1 & -5 & $(1)$ & 10.9 & 65 \\
& -3 & 220 & $(2)$ & & \\
39750 & 0 & 0 & $(1)$ & 11.5 & 70 \\
& -2 & 30 & $(2)$ & & \\
40880 & 0 & 0 & $(2)$ & 10.9 & 40 \\
& -4 & 319 & $(3)$ & & \\
41702 & 1 & 242 & $(2)$ & 11.7 & 25 \\
& -3 & 111 & $(3)$ & & \\
42455 & 2 & 415 & $(2)$ & 10.6 & 60 \\
& -2 & -166 & $(3)$ & & \\
43697 & -1 & 46 & $(3)$ & 12.3 & 20 \\
44681 & 0 & 0 & $(3)$ & 10.6 & 60 \\
45559 & 1 & -152 & $(3)$ & 10.4 & 67 \\
46776 & 2 & 35 & $(3)$ & 10.4 & 76 \\
47759 & 3 & -12 & $(3)$ & 10.4 & $<71$ \\
48834 & 4 & 33 & $(3)$ & 10.4 & $>66$ \\
50170 & 5 & 339 & $(3)$ & 10.4 & 65 \\
51264 & 6 & 403 & $(3)$ & 10.4 & 73 \\
\hline & & & & &
\end{tabular}



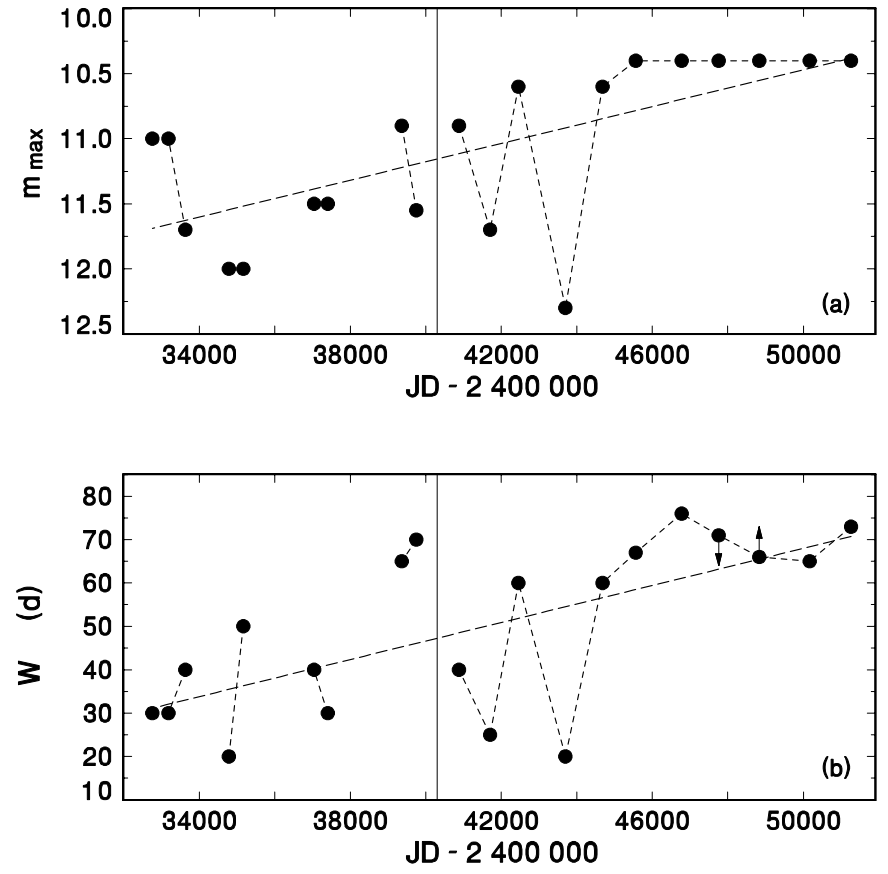

Fig. 2. Variations of $m_{\max }$ and $W$ of the outbursts in GK Per as a function of the Julian Date. Consecutive maxima (Sect. 3.4) are connected by a line. Both $m_{\max }$ and $W$ generally tend to increase, as shown by the linear fits. The points with arrows mark the upper or lower limits of $W$ and were not included in the fits. The vertical line denotes the moment of a large jump of $T_{\mathrm{C}}$. See Sect. 3.2 for details.

peak can be constrained to within about 20 days, that is much less than the recurrence time $T_{\mathrm{C}}$.

\subsection{The outburst parameters in the optical region}

The width $W$ and $m_{\max }$ of the outbursts in GK Per allow a reliable analysis of the energy output of each event. Both these parameters were either taken from Table 3 in Sabbadin \& Bianchini (1983) or measured in the AFOEV data. The outburst width $W$ refers to the duration of the event, measured with respect to the quiescence level. The AFOEV data also enabled us to re-measure and slightly correct $m_{\max }$ and $W$ for some events, listed by Sabbadin \& Bianchini.

Variations of $m_{\max }$ and $W$ are displayed as a function of the Julian Date in Fig. 2. Both these parameters display very prominent changes. One also can readily resolve some trends. The maximum brightness varies by more than $1.5 \mathrm{~m}_{\mathrm{v}}$ and displays a general positive trend. Finally, $m_{\max }$ seems to stabilize at $10.4 \pm 0.1 m_{\mathrm{v}}$ after JD 2445000 . Also $W$ tends to rise, although the scatter is large, sometimes due to the tendency of the short and long outbursts to alternate.

The relation between $m_{\max }$ and $W$ can be assessed from Fig. 3. The least squares fit confirms that there is a clear tendency for the fainter outbursts to be shorter and

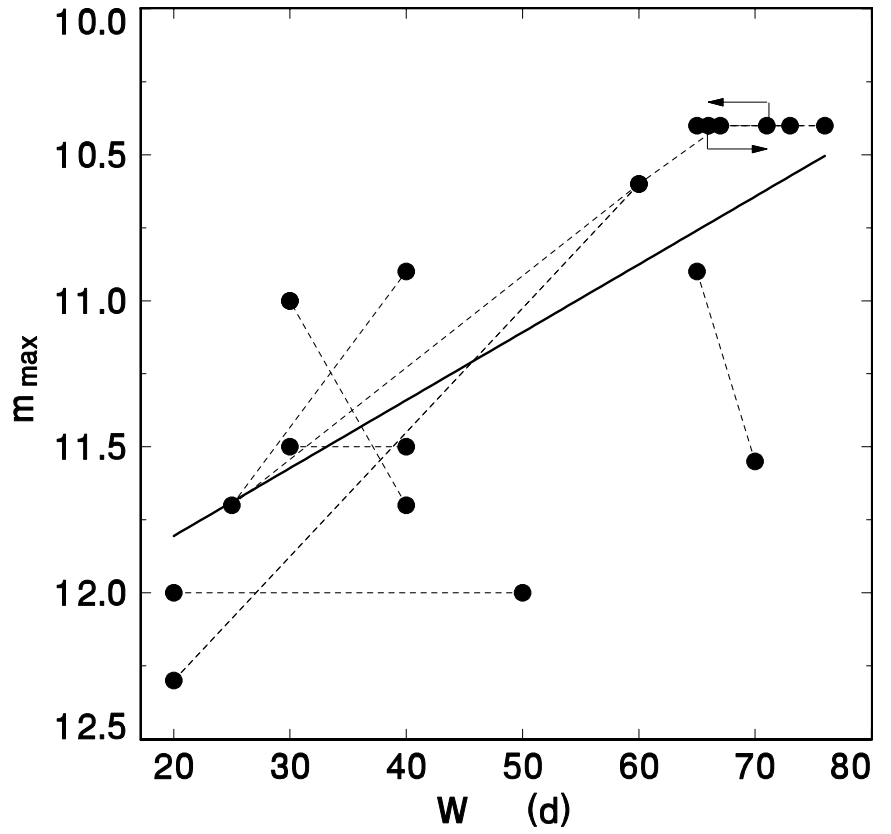

Fig. 3. Correlation between $m_{\max }$ and the outburst width $W$ in GK Per. Consecutive maxima are connected by a line. The least squares fit is marked by the thick solid line. The points with arrows represent the upper or lower limits of $W$ and were not included in the fits. See Sect. 3.2 for details.

vice versa. It implies that the brighter outbursts usually have also a larger energy in the optical region.

\subsection{ASM/RXTE observations of outbursts}

The outbursts of GK Per in the years 1996 and 1999 were covered by the $A S M / R X T E$. Although in both cases the signal was weak, even at light maximum an attempt to roughly determine the X-ray light curve was made. $A S M / R X T E$ data cover the whole duration of the outbursts and so they give a unique opportunity to compare the behaviour in the hard X-ray and optical regions. In order to lower the noise, only daily means with uncertainty smaller than 0.6 count/s were used for the analysis. We note that the rapid day-to-day fluctuations in Figs. 4b,d are likely to be mainly caused by the observational noise. The data were therefore carefully smoothed by several methods. Firstly, the code HEC13, written by Dr. P. Harmanec and based on the method of Vondrák (1969; 1977), was applied. This method can fit a smooth curve no matter what the course of the data is. After several trials the input parameters of the fit $\epsilon=10^{-1}$, the length of the bin $\Delta T=20$ days were found to satisfy the course of the data. The $\epsilon$ parameter determines how "tight" the fit will be, that is if just the main course or also the high-frequency variations are to be reproduced. Given the large noise of the $A S M$ observations of GK Per, the final choice of $\epsilon$ intended to reproduce only the main course of the curve. Secondly, the two-sided moving averages were calculated for $Q=10,12$ and 15 days. Here the values of $Q$ refer to the semi-interval of days, within 

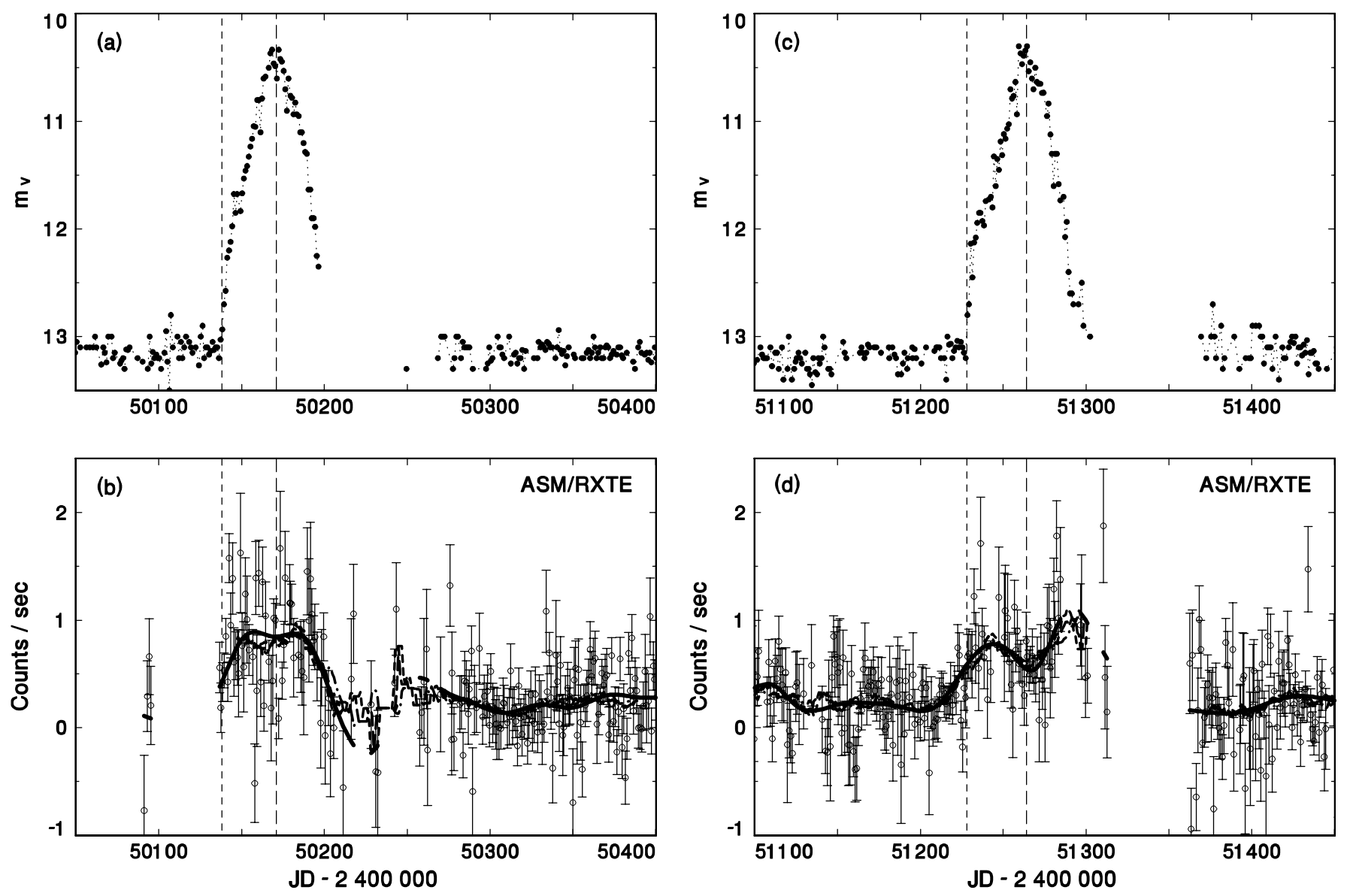

Fig. 4. Relation between the outburst light curves of GK Per in the optical and hard X-ray (2-12 keV) passbands. Both the optical and the X-ray observations were binned into one-day means. The quoted uncertainties of the $A S M / R X T E$ data are included. The thick lines in $\mathbf{b}, \mathbf{d}$ ) denote the smoothing by the code HEC13 (solid line) and the two-sided moving averages, calculated for $Q=10$ days (short-dashed line), $Q=12$ days (medium-dashed line) and $Q=15$ days (dot-dashed line). The dashed vertical lines mark the moments of the onset and the maximum light of the outburst in the optical region. See Sect. 3.3 for details.

which the data were averaged. The X-ray light curves of the two outbursts are shown in Fig. 4. The optical light curves are shown for comparison. The dashed vertical lines mark the moments of the onset and of the light maximum in the optical region and enable a comparison with the behaviour in the X-rays. The fits to $A S M$ data by both methods are in good agreement and reveal that the onset of the outburst can be considered as roughly simultaneous in the optical and the X-rays. The outbursts have clearly flat-topped maxima in the hard X-rays, with a depression apparent in the later event.

\subsection{The outburst recurrence time and its variations}

The method of the $\mathrm{O}-\mathrm{C}$ residuals from some reference period (e.g. Vogt 1980) enables us to determine $T_{\mathrm{C}}$ in a dwarf nova and to analyze its variations. This method is not sensitive to the exact length of the reference period. $\mathrm{O}-\mathrm{C}$ diagram can be constructed even if there are gaps in the data and hence some missed outbursts. A more detailed discussion of this method was given by Šimon (2000a).
The statement by Bianchini et al. (1986) that $T_{\mathrm{C}}$ in GK Per may be considered as a multiple of $400 \pm 40$ days was used as a starting guideline in the search for the length and variations of $T_{\mathrm{C}}$. A careful examination of the $\mathrm{O}-\mathrm{C}$ diagrams, generated for various $T_{\mathrm{C}}$ 's and the $T_{0}$ epochs in order to minimize the mean slope of the $\mathrm{O}-\mathrm{C}$ values, revealed that there are basically two intervals with quite different recurrence times. The variations of $T_{\mathrm{C}}$ in GK Per were found to be so large that they cannot be displayed in a single $\mathrm{O}-\mathrm{C}$ diagram. The values of $T_{\mathrm{C}}$ 's, used in the resulting ephemerides (Eqs. (1)-(3)), have to be very divergent to keep a low slope of the $\mathrm{O}-\mathrm{C}$ curve. The $\mathrm{O}-\mathrm{C}$ diagram then becomes distorted in some segments because some outbursts fall into the same calculated epoch $E$. In order to overcome this, the $\mathrm{O}-\mathrm{C}$ curves were generated for the data just inside the time intervals within which the distortions of the course of the $\mathrm{O}-\mathrm{C}$ values could be avoided.

$$
\begin{aligned}
& T_{\max }=2439750+385 E \\
& T_{\max }=2440880+580 E \\
& T_{\max }=2444681+1030 E
\end{aligned}
$$



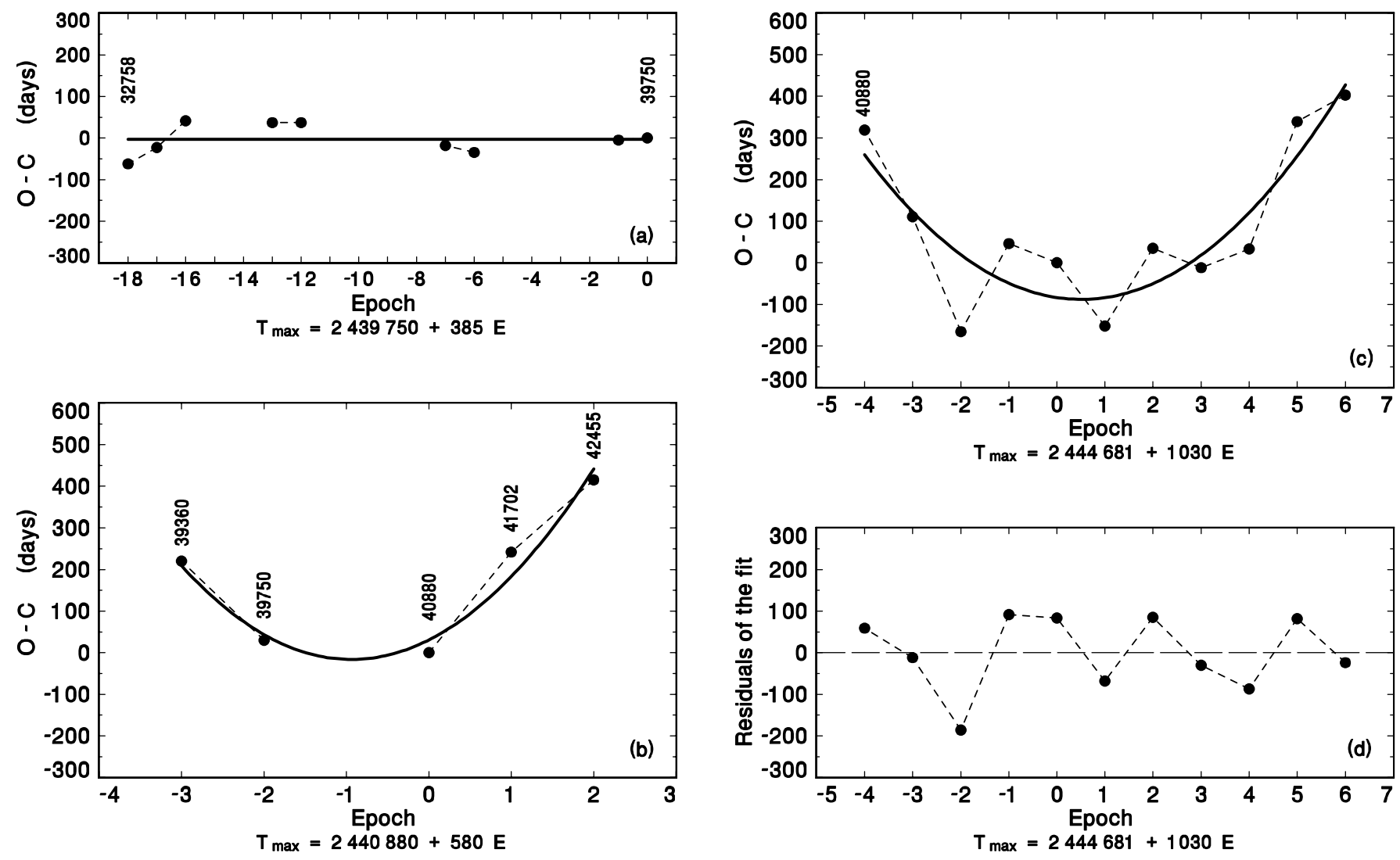

Fig. 5. O-C diagram for the moments of the outburst maxima in GK Per. The variations of $T_{\mathrm{C}}$ are so large that they cannot be displayed in a single diagram. The $\mathrm{O}-\mathrm{C}$ values in the respective plots were calculated according to Eqs. (1)-(3). The scales of the vertical axes are identical for all four plots. The error bars of the respective $\mathrm{O}-\mathrm{C}$ values are smaller than or comparable to the size of the symbols. Consecutive outbursts are connected by a dashed line. The thick lines denote the linear or quadratic fits to the segments of the $\mathrm{O}-\mathrm{C}$ curve. The Julian Dates, labeled for some points, allow an easier orientation in the plots. d) shows the residuals of the quadratic fit from c). See Sect. 3.4 for details.

The outbursts in the interval JD 2432 758-2439750 can be folded with $T_{\mathrm{C}}=385$ days (Eq. (1)). The course of the $\mathrm{O}-\mathrm{C}$ values can be considered as linear (i.e. $T_{\mathrm{C}}$ is constant) with the standard deviation $\sigma=36$ days (Fig. 5a). However, a dramatic change of $T_{\mathrm{C}}$ occurred around JD 2440880 ; the recurrence time rapidly increased and the $\mathrm{O}-\mathrm{C}$ diagram began to display a strong parabolic trend with superposed smaller but real cycle-to-cycle variations (Fig. 5c). There is just a very little difference between the fit by the 2nd and the 3rd order polynomial, the standard deviations of the residuals being 90 and 89 days, respectively. The fluctuations of the residuals of the quadratic fit in Fig. $5 \mathrm{~d}$ are real because in most cases the error bars in Fig. 5 would be smaller than or comparable to the size of the symbols used. Examination of the residuals of the quadratic fit from Fig. 5d by the code SPEL, written by Dr. J. Horn and based on the method of Stellingwerf (1978), revealed that there may be present cyclic variations with the cycle-length of $2.92 \pm 0.07$ epochs and semi-amplitude of $115 \pm 20$ days. However, since the number of outbursts inside this interval is still relatively small it would be premature to draw strong conclusions from this cycle.
It is apparent from Fig. 5a that outbursts at some epochs are absent. However, a comparison with the plots of the light curves in Sabbadin \& Bianchini (1983) confirmed that several outbursts could really be missed because there are long intervals not covered by the observations. The moments of all outbursts, missing in Fig. 5a, were calculated according to Eq. (1) and compared with Fig. 4 of Sabbadin \& Bianchini (1983). This procedure confirmed that all these but one missing outbursts really fall into intervals of the light curve where the coverage is quite weak or the data are even missing. The only exception may be the outburst at $E=-15$; its calculated position coincides with a broad bump of slightly increased brightness level with some superposed fluctuations. There are also two relatively long seasonal gaps surrounding this bump, starting at approx. 120 and 160 days from the calculated position.

On the other hand, there is good reason to believe that no outburst is missing in Fig. 5c. The seasonal gaps after JD 2445500 are short (initially 80-90 days, later 30-60 days) (Fig. 1) and there is an observed outburst for every epoch, calculated according to Eq. (3). 


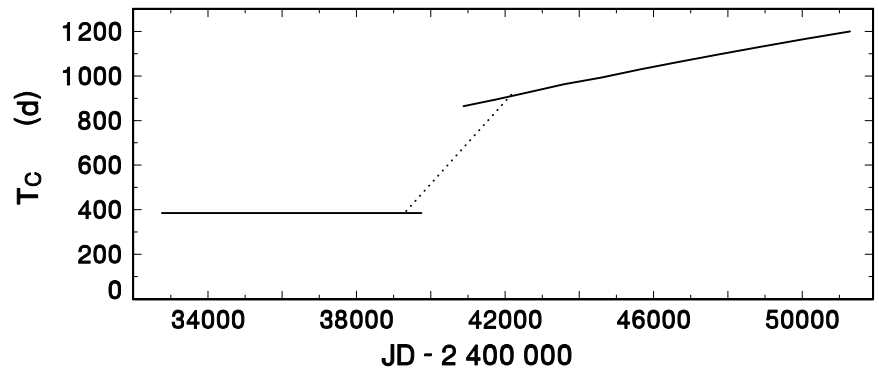

Fig. 6. Evolution of $T_{\mathrm{C}}$ (in days) of the outbursts in GK Per. Notice the large jump in $T_{\mathrm{C}}$ around JD 2441 000. See Sect. 3.4 for details.

In order to show the huge change of $T_{\mathrm{C}}$ around JD 2440880 , one more $\mathrm{O}-\mathrm{C}$ diagram was generated using Eq. (2) (Fig. 5b). This ephemeris was adjusted so that the $\mathrm{O}-\mathrm{C}$ curve was undisturbed for at least several outbursts. There are two possible interpretations: (a) rapid abrupt change within a single cycle (the absent outburst at $E=-1$ would fall into a gap in Sabbadin \& Bianchini's (1983) data) or (b) parabolic change with a very rapid increase of $T_{\mathrm{C}}$.

In summary, it can be seen that the method of residuals enabled us to determine $T_{\mathrm{C}}$ and its large variations in GK Per with certainty in spite of several missing outbursts which fall into intervals of a poor coverage of the light curve.

Figure 6 shows the evolution of $T_{\mathrm{C}}$, determined from the $\mathrm{O}-\mathrm{C}$ diagrams. Notice the large jump in $T_{\mathrm{C}}$ around JD $2441000 . T_{\mathrm{C}}$ more than doubled within perhaps a single epoch and then steadily increased. The dotted line denotes the alternative evolution of $T_{\mathrm{C}}$, determined from the fit to the $\mathrm{O}-\mathrm{C}$ values in Fig. $5 \mathrm{c}$.

\subsection{Morphology of the outburst light curves}

The outbursts of GK Per, displayed in Fig. 1, are characteristic for the time interval of gradually increasing mean recurrence time after the jump of $T_{\mathrm{C}}$. They allow us to resolve unambiguously the features on their light curves because they are quite wide. Again, the procedure used here was similar to that applied to $\mathrm{CH}$ UMa by Šimon (2000a). Firstly, we analyzed the properties of the decay branches. The outburst having maximum in JD 2451264 was chosen as the template because its decay branch is well covered. The remaining outbursts from Fig. 1 were shifted along the time axis to match the decay branch of the template. The level of brightness of $11.2 \mathrm{~m}_{\mathrm{v}}$ was chosen as the reference level, in the vicinity of which the match was attempted. However, it should be stressed that the decay branches of these outbursts were found to be so similar each to other that the quality of the match was to a large extent independent of the choice of the reference level. The result is shown in Fig. 7. The decay branches of the respective outbursts were then merged into a common file and smoothed by the above-mentioned code HEC13. $\epsilon=10^{-1}$, $\Delta T=5$ days were found to satisfy the main course of the decay. The standard deviation of the residuals of this fit is just $0.15 m_{\mathrm{v}}$ and remains essentially constant over the whole decay. The smoothed decay light curve is plotted as the thick solid line in Fig. 7. Notice also that there is a well defined tail in the final phase of the decay, starting at $12.6 \mathrm{~m}_{\mathrm{v}}$.

The models predict that the decay time scale $\tau_{\mathrm{D}}$, expressed in days $m_{\mathrm{v}}^{-1}$, can be influenced by the magnetic field of the WD (Angelini \& Verbunt 1989; Cannizzo 1994). Comparison of GK Per with other dwarf novae is shown in Fig. 8 where $\tau_{\mathrm{D}}$ is plotted versus orbital period $P_{\text {orb }}$ (so called Bailey relation, Bailey 1975). The data for most non-magnetic systems (solid points) and two possible magnetic systems SW UMa and WZ Sge come from Warner (1995) while those for DX And come from Šimon (2000c). The solid line denotes the fit to non-magnetic systems with $P_{\text {orb }}<15 \mathrm{hr}$ while the dashed line marks its extrapolation. The fit by the dotted line includes also V 1017 Sgr. The magnetic CVs DO Dra (Šimon 2000b) and WZ Sge (Lasota et al. 1999) lie well below the nonmagnetic CVs in Fig. 8. On the other hand, GK Per falls close to Bailey relation. Two fits are shown: the whole decay, whose starting point is defined by the beginning of the fit in Fig. 7 , yields $\tau_{\mathrm{D}}=14.5$ days $m_{\mathrm{v}}^{-1}$ no matter if the tail is included or not; the steepest part, marked by the straight line in Fig. 7 , yields $\tau_{\mathrm{D}}=8.0$ days $m_{\mathrm{v}}^{-1}$. They are plotted as a triangle and a box in Fig. 8 .

A similar approach as for the decays was used also to analyze the rising branches of the outbursts (Fig. 9). Again, the respective outbursts were shifted along the time axis to match the template. The rising branches of the individual events were found to be significantly more variable than the decays. In order to make the problem tractable, it was decided to match the initial parts of the rising branches within 12.2 and $13.2 \mathrm{~m}_{\mathrm{v}}$. The brightness of $12.5 m_{\mathrm{v}}$ was chosen as the reference level. This procedure artificially minimized the scatter here but it showed that the largest variations occur in the middle part of the rise. The respective curves then converge each to the other again as they approach the peak brightness.

\subsection{Long-term variations of the quiescent level}

Variations of the quiescent level of brightness in GK Per were searched for in the AFOEV data after removing the outbursts. Only analysis of the long-term trends on the time scale of years is meaningful in the visual data. Moving averages were used to suppress the high frequency variations, generally caused by both the observational noise and the real rapid changes, and to pick out the general trends. This method allowed Cannizzo \& Mattei (1992) to resolve variations of the quiescent level of SS Cyg with an amplitude of just $0.1 \mathrm{mag}$ in the visual data.

Two-sided moving averages were calculated for $Q=$ 400, 500, 700 and 1000 days (Fig. 10a). The respective fits are in good agreement and confirm that the full amplitude 


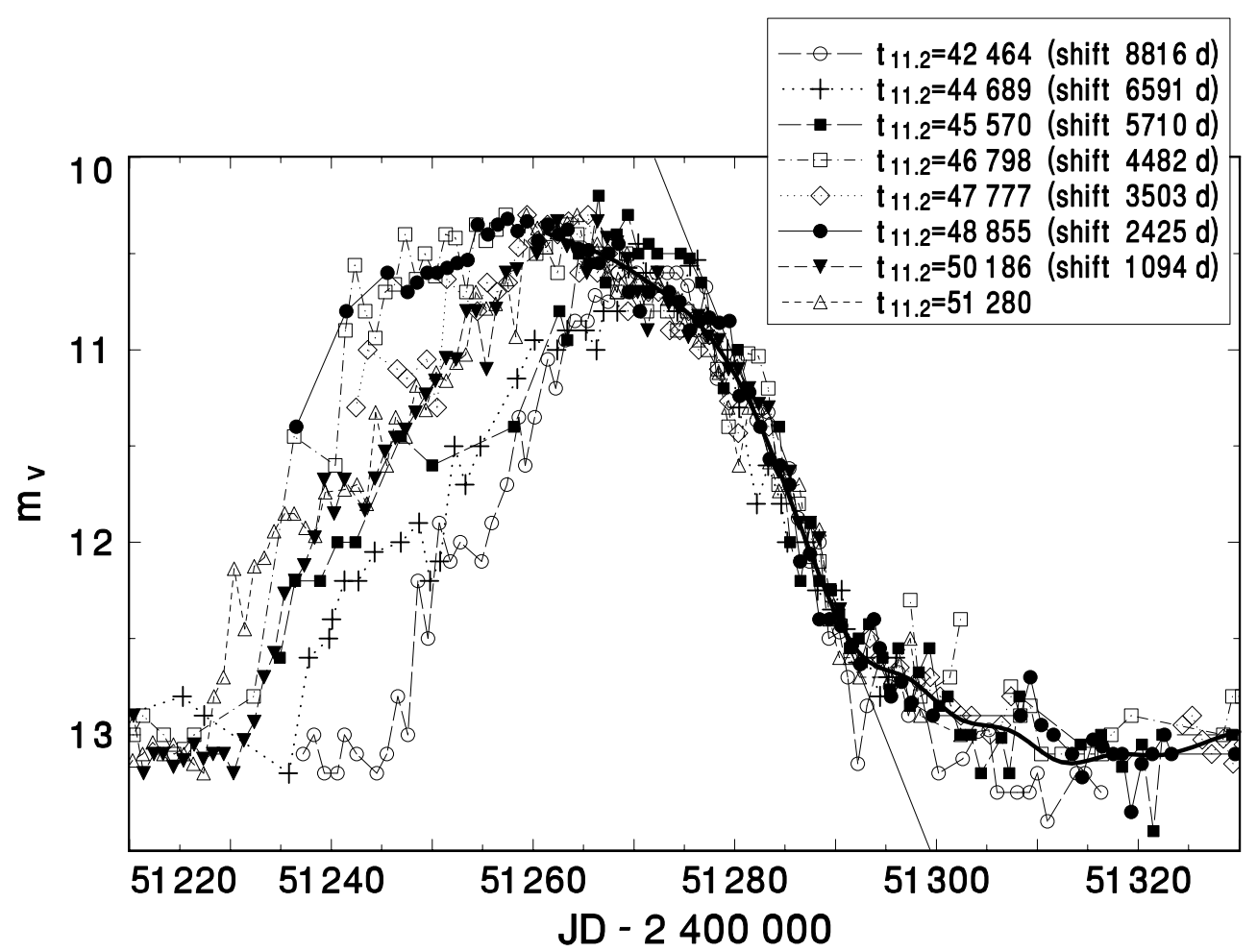

Fig. 7. The profiles of the well covered outbursts in GK Per, based on the AFOEV observations and supplemented by the data of Szkody et al. (1985) when necessary. The points represent one-day means and were connected by a line in densely covered parts of the light curves. The respective outbursts were shifted along the time axis to match the decay branch of the template the time of crossing $11.2 m_{\mathrm{v}}$ and the shifts with respect to the template are listed in the figure. The thick curve represents the smoothed decay curve. The tilted line denotes the part of the decay branch which can be considered as exponential. See Sect. 3.5 for details.

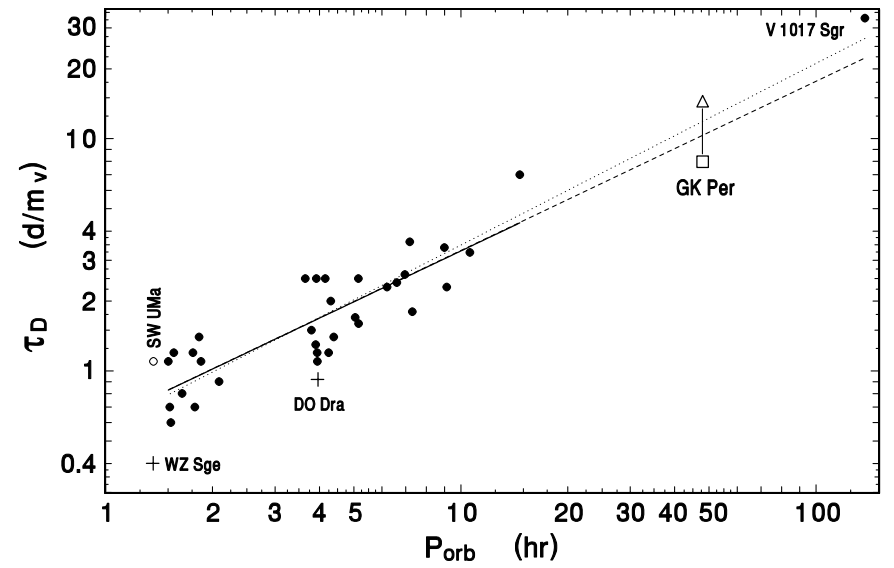

Fig. 8. Decay time scale of dwarf nova outbursts $\tau_{\mathrm{D}}$ versus orbital period $P_{\text {orb }}$. The data for non-magnetic systems (solid points) come mostly from Warner (1995). The solid line marks the fit to non-magnetic systems with $P_{\text {orb }}<15 \mathrm{hr}$ while dashed line marks its extrapolation. The fit by dashed line includes also V 1017 Sgr. Magnetic or suspected systems are denoted by + and $\circ$, respectively. Two fits for GK Per are plotted. See Sect. 3.5 for details.

of the variations of the quiescent level amounts to approx. $0.3 m_{\mathrm{v}}$. The course of variations can be described as a brightening till JD 2447000 and a smooth decrease after this date. There is an indication that the decrease stopped at JD 2450500 . The smoothed light curve thus resembles a wave on the time scale of about 30 years.

\section{Discussion}

Our analysis has brought the lines of evidence that the intermediate polar GK Per experienced a very striking evolution of both the outburst energetics and the recurrence times after the year 1948 .

The character of the $\mathrm{O}-\mathrm{C}$ curves for $T_{\mathrm{C}}$ of the dwarf nova-type outbursts (Fig. 5) bears a large similarity to other dwarf novae (Vogt 1980; Šimon 2000a,b,c), i.e. variations of $T_{\mathrm{C}}$ are large but generally not chaotic. Figure 5 displays beyond any doubt that long-term trends can be resolved in the $\mathrm{O}-\mathrm{C}$ curves. The $\mathrm{O}-\mathrm{C}$ diagrams display $T_{\mathrm{C}}=385$ days within the years 1948-1967. The previous finding that $T_{\mathrm{C}}$ in GK Per is a multiple of $400 \pm 40$ days (Bianchini et al. 1986; Hudec 1981) is therefore valid only in this interval. The evolution of $T_{\mathrm{C}}$ then shows a large jump. $T_{\mathrm{C}}$ more than doubled within perhaps just one single epoch and then steadily increased. It is about 1230 days at present. Even if we accept the interpretation that $T_{\mathrm{C}}$ evolved gradually around JD 2441000 the discontinuity in Fig. 6 is still very prominent. It is interesting to note that the residuals of the parabolic fit (Fig. 5d) display a larger scatter than the residuals of the linear ephemeris in Fig. 5a. The ratio of the mean $T_{\mathrm{C}}$ 's in Figs. $5 \mathrm{a}$ and $5 \mathrm{c}$ 


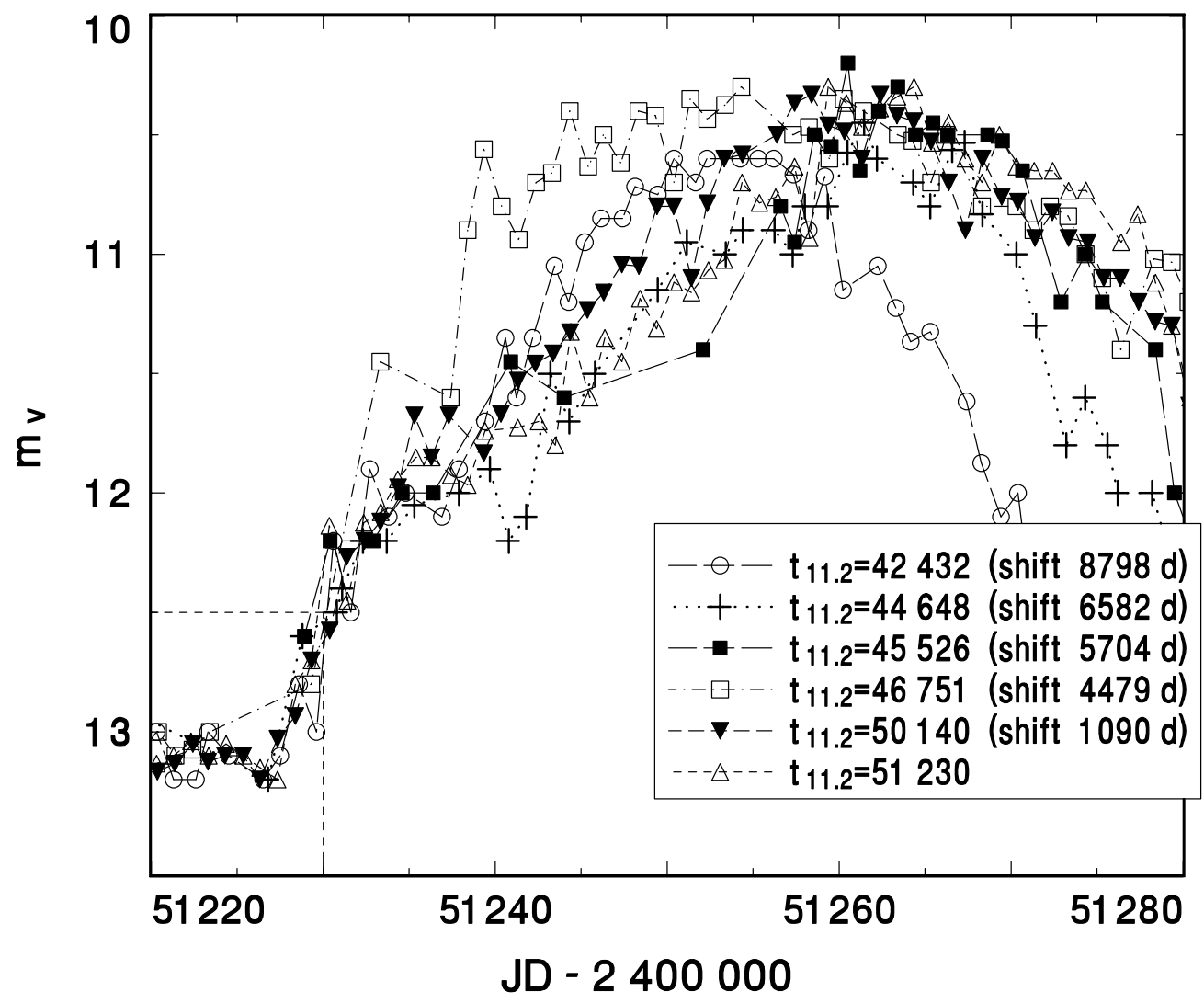

Fig. 9. The rising branches of the outbursts in GK Per from Fig. 7. Only outbursts with the covered initial part of the rise were analyzed. The individual outbursts were shifted along the time axis to match the bottom part of the rising branch of the template. The time of crossing $12.5 \mathrm{~m}_{\mathrm{v}}$ and the shifts with respect to the template are listed in the figure. See Sect. 3.5 for details.
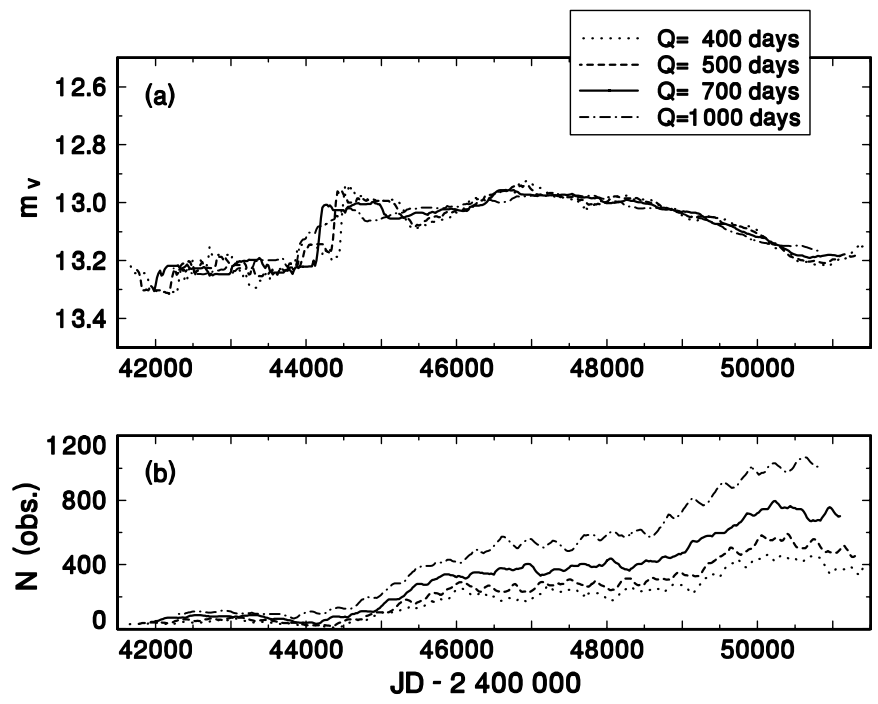

Fig. 10. Two-sided moving averages of the quiescent level of brightness of GK Per a) and the number of observations inherent in each mean b). Four curves for different $Q$ are shown to confirm the course of the light curve. See Sect. 3.6 for details.

is about $1: 2.7$. It is therefore tempting to infer that the scatter of the residuals increases with the length of the average $T_{\mathrm{C}}$. Alternatively, the larger scatter in the latter case might be attributed to the cyclic term.

Strong evidence emerged that variations of $T_{\mathrm{C}}$ in GK Per are related to the changes of $m_{\max }$ and $W$. The increase of $T_{\mathrm{C}}$ was accompanied by a brightening of $m_{\max }$ and increase of $W$. Both $m_{\max }$ and $W$ then stabilized within a few epochs and remained at the values significantly different from those before the jump of $T_{\mathrm{C}}$. Brighter $m_{\max }$ at longer $W$ implies that the total energy of the outbursts, radiated in the optical passband, increases. Since there is an apparent evolution of activity after the nova explosion in 1901, from irregular fluctuations (Sabbadin \& Bianchini 1983) through (semi)regular outbursts of a larger amplitude to even more energetic, but less frequent outbursts, it is tempting to interpret these changes as a consequence of a large increase of the temperature of the WD and of the irradiation of the secondary.

The secondary star becomes strongly irradiated during the nova explosion and slowly cools down afterwards, so the mass transfer rate $\dot{m}$ is enhanced in the next decades. However, a pure decrease of $\dot{m}$ cannot account for the observed increase of $T_{\mathrm{C}}$ of GK Per in the framework of the latest models for the thermal instability (TI) (Hameury et al. 1998). They predict that the decrease of $\dot{m}$ leads to an increase of $T_{\mathrm{C}}$, but at the same time also to a decrease 
of the outburst energy. The reverse is true for GK Per, so the effect of decreasing $\dot{m}$ does not appear to be dominant.

The WD remains very hot $\left(T_{\text {eff }}\right.$ a few $\left.\times 10^{5} \mathrm{~K}\right)$ after the nova explosion and its luminosity decreases with time as $L \propto t^{-1.14}$ (Prialnik 1986). Irradiation of the disk by the WD can substantially alter its structure and several scenarios can be offered in the framework of the models for TI. If a given disk is thermally unstable in the absence of irradiation then a strong irradiation by the WD can suppress TI completely while a weaker one gives rise to an inner steady-state disk region, surrounded by a thermally unstable outer annulus. Several models (Mineshige et al. 1990; Schreiber et al. 2000) confirmed that TI can operate in this annulus. They agree that $T_{\mathrm{C}}$ is shorter than in the absence of irradiation and just $m_{\min }$, and not $m_{\max }$, change with irradiation. According to the model by Leach et al. (1999), the heating front may not propagate all the way to the outer rim of irradiated disk, so just a series of short outbursts appears. These models are to some extent in accordance with the observed behaviour of GK Per if we suppose that TI operated in the outer annulus of the irradiated disk in the period 1948-1967. Later, TI could spread over the whole disk as the further cooling WD brought the whole disk into a thermally unstable regime. Also the reddening of $B-V$ in quiescence from the fifties to 1989 is compatible with this scenario $(B-V=0.71$ : Walker 1957; $B-V=0.87$ : Mumford 1966; $B-V=0.90$ to 0.95 : Wu et al. 1989). However, some problems remain. While in another old nova V 446 Her the brightness prior to the interval in which the outbursts appeared was comparable to $m_{\max }$ (Schreiber et al. 2000), the level of brightness in GK Per prior to 1948 was closer to its minimum level. This problem may possibly be reconciled if some additional effects are included, like variations of the mass and angular momentum transport through the disk. For example $T_{\mathrm{C}}$ in a given $\mathrm{CV}$ is inversely proportional to the quiescent viscosity parameter $\alpha_{\text {cool }}$ (e.g. Warner 1995). If $\alpha_{\text {cool }}$ is allowed to have a lower value after the jump of $T_{\mathrm{C}}$ than before it then a larger amount of matter can accumulate in the disk during a longer quiescent interval. This can then power a brighter outburst. However, it is difficult to separate both effects (irradiation and variations of $\left.\alpha_{\text {cool }}\right)$ unambiguously in GK Per.

The faint outburst at JD 2443697 shows that although this event is much weaker in the total energy, radiated in the optical region, than most others after the jump of $T_{\mathrm{C}}$, it fits well into the mean course of the $\mathrm{O}-\mathrm{C}$ diagram. This event was accompanied by an X-ray brightening (King et al. 1979). This suggests that an accretion onto the WD occurred, so it was not just an increase of brightness of the hot spot, caused by a mass transfer burst. This suggests that although the TI was stopped in its early phase and full outburst did not develop it did not affect the cyclelength to the following outburst.

The decay branches of outbursts in Fig. 7, that is those which occurred after the jump of $T_{\mathrm{C}}$, remain remarkably similar for the individual events. This suggests that all the decays are controlled by the same process with very similar parameters. In the framework of the model for TI (e.g. Smak 1984), the decay is governed by the cooling front which always starts in the outer part of the disk and moves inwards. The similarity of the decays (Fig. 7) then suggests that the properties of this front remain stable for the respective events. It allows us to infer that both the inner and outer disk radius $r_{\text {in }}$ and $r_{\text {out }}$, respectively, attain quite a similar value at the end of each outburst - the model predicts that $\tau_{\mathrm{D}}$ is a function of both $r_{\mathrm{in}}$ and $r_{\text {out }}$ (Cannizzo 1994). Especially $r_{\text {in }}$ plays an important role in intermediate polars because it is governed by the strength of the magnetic field of the WD. Nevertheless, the influence of this field on the decay branch in GK Per seems to be rather weak. The position of GK Per is not markedly different from the $\tau_{\mathrm{D}}$ vs. $P_{\text {orb }}$ relation for non-magnetic systems (Fig. 8) although according to Cannizzo's (1994) TI model the decay becomes faster and ceases to be exponential with increasing $r_{\text {in }}$. Even if just the part of the decay with the steepest slope is taken, the difference between GK Per and the fit in Fig. 8 is still smaller than in the case of another intermediate polar DO Dra. It can suggest that the true $r_{\text {in }}$ in GK Per is not as large as inferred by Kim et al. (1992) TI models which predict $r_{\text {in }}$ approx. four times larger than the corotation radius $r_{\text {cor. }}$. Also the QPO favour a smaller $r_{\text {in }} \approx 1 r_{\text {cor }}$ (Morales-Rueda et al. 1999). In addition, the decrease of the QPO cycle-length with the progress of outburst may suggest a decrease of $r_{\text {in }}$, so $\tau_{\mathrm{D}}$ can become comparable to non-magnetic $\mathrm{CVs}$.

The shape of the late decay branch of outbursts in GK Per is more complicated than usual for other dwarf novae. Such outbursts usually finish with a rapid transition from the decay to the quiescent level while a tail can be resolved in GK Per. A very similar feature was observed in some outbursts of SS Cyg (Cannizzo \& Mattei 1998) and in the long-period system CH UMa (Šimon 2000a), but only in a class of outbursts with exceptionally long decay throughout. On the contrary, the tail appears to be common in GK Per. This feature may be tentatively explained by irradiation of the disk by the WD because re-flares are present in some models (Hameury et al. 2000).

The middle part of the rising branch is the interval of the light curve which undergoes the largest changes for the respective outbursts in GK Per (Fig. 9). This phenomenon can be explained in the framework of the TI model in the following way. In the case that the onset of TI occurs in the outer part of the disk the outburst is of type A (outside-in). Its light curve has a rapid rise and slow decay. The TI of the B type outburst, starting in the inner parts of the disk and propagating outwards (inside-out), produces instead symmetric light curve with a slow rise. The B type is typical for systems with a lower mass transfer rate $\dot{m}$ than A type (Smak 1984). The location of the onset of TI can depend also on the drift of matter during quiescence, governed by $\alpha_{\text {cool }}$ and the position where matter from the stream feeds the disk (e.g. stream overflow). In the framework of TI, Fig. 9 can be then interpreted that the heating front starts at a different radial distance from the center of the disk for the respective events. 
The further from the center of the disk the onset of TI occurs, the more the outburst resembles type A. The TI models predict that there is no difference between the decay of type A and B outburst, in accordance with Fig. 7. A similar behaviour was observed also in another longperiod dwarf nova DX And $\left(P_{\text {orb }}=10.6 \mathrm{hr}\right.$ ) (Šimon 2000c) but it seems that generally the properties of the rise differ from system to system: a very uniform shape of the vast majority of the rise branches was found in SS Cyg $\left(P_{\text {orb }}=6.6 \mathrm{hr}\right)$ (Cannizzo \& Mattei 1998). Possibly an analysis of a large sample of systems with various $P_{\text {orb }}$ may help clarifying this phenomenon.

$A S M / R X T E$ observations of two outbursts showed useful features after a careful smoothing. The onset and the decay can be considered as roughly simultaneous in the optical and the hard X-rays but the profiles of the light curve in these two spectral regions are discordant. While the optical light curves have a sharp top, clearly flat-topped maxima are apparent in the hard X-rays, with a possible depression coincident with the moment of the optical maximum in the 1999 event. This behaviour bears some resemblance to SS Cyg (Jones \& Watson 1992). Previous X-ray observations of GK Per were interpreted in terms of a lower absorption in quiescence than in outburst (Norton et al. 1988). The flat profile of the $A S M / R X T E$ light curve of both events and its central depression during the optical maximum of 1999 may be then attributed to the blocking of hard X-rays by the thickened disk. In this time, just the component of the hard X-ray emission, scattered in the accretion column, may be observable (Yi et al. 1992). Another process is the radiation drag which becomes important when $\dot{m}$ exceeds some critical value during the rise of the outburst; the emitted energy in the $A S M / R X T E$ passband then begins to decrease despite further rising $\dot{m}$ (Yi \& Vishniac 1994, their Fig. 5). The models by Kim et al. (1992) predict that a brightening in $\mathrm{X}$ ray and UV can precede the optical outbursts in GK Per by $80-120$ days. Such a precursor is absent in the 1999 event but it seems to have occurred in the much fainter and shorter 1978 outburst (Bianchini \& Sabbadin 1985).

Variations of the quiescent level of GK Per, detected during the last about 30 years, did not show any secular trend after the nova explosion. Instead, they can be described as a wave on the time scale of about 30 years. Similar variations were suggested also by Richman et al. (1994) but now the fit shows them with the best clarity. Figure 10 shows no sign of the short-term variations ( $\sim 7$ years) and the full amplitude $0.3 \mathrm{mag}$, suggested to be present within the years 1920-1986 and attributed to the solar-type cycle by Bianchini (1990). Such rapid changes must have completely disappeared at latest around JD 2446000 . Our analysis further revealed that the changes of the quiescent level are not correlated with variation of $T_{\mathrm{C}}, m_{\max }$ or $W$. The disk is dim in quiescence, so the K-type secondary contributes significantly to the total luminosity $(1 / 3$ to $1 / 2$ : Wu et al. 1989; Anupama \& Prabhu 1993). The interpretation of the observed wave in terms of luminosity variations of the secondary is therefore reasonable because variations of the mean photospheric temperature by just a few hundreds of Kelvins, caused for example by a variable coverage by the dark (cool) spots are then sufficient to invoke changes of the system luminosity of the observed amplitude. The evidence for the cool star activity is supported also by the presence of the strong chromospheric line of Mg II $\lambda \lambda$ 2796-2802 (Rosino et al. 1982).

Acknowledgements. This research has made use of NASA's Astrophysics Data System Abstract Service, the AFOEV database, operated at CDS, France, and the observations provided by the ASM/RXTE team. I thank Dr. Hudec for reading the manuscript and for the comments. I am also indebted to Dr. Harmanec for providing me with the program HEC13. Naturally, my thanks also to numerous amateur observers worldwide whose observations made this analysis possible. The support by the post-doctoral grant 205/00/P013 of the Grant Agency of the Czech Republic and the project ESA PRODEX INTEGRAL 14527 is acknowledged.

\section{References}

Anupama, G. C., \& Prabhu, T. P. 1993, MNRAS, 263, 335

Angelini, L., \& Verbunt, F. 1989, MNRAS, 238, 697

Bailey, J. 1975, J. Brit. Astr. Ass., 86, 30

Bianchini, A. 1990, AJ, 99, 1941

Bianchini, A., \& Sabbadin, F. 1985, IBVS, 2751

Bianchini, A., Sabbadin, F., Favero, G. C., \& Dalmeri, I. 1986, A\&A, 367, 373

Cannizzo, J. K. 1994, ApJ, 435, 389

Cannizzo, J. K., \& Mattei, J. A. 1992, ApJ, 401, 642

Cannizzo, J. K., \& Mattei, J. A. 1998, ApJ, 505, 344

Crampton, D., Cowley, A. P., \& Fisher, W. A. 1986, ApJ, 300, 788

Duschl, W. J., Meyer-Hofmeister, E., \& Meyer, F. 1985, ESA Recent Results on Cataclysmic Variables, 221

Hameury, J.-M., Menou, K., Dubus, G., Lasota, J.-P., \& Huré, J.-M. 1998, MNRAS, 298, 1048

Hameury, J.-M., Lasota, J.-P., \& Warner, B. 2000, A\&A, 353, 244

Hudec, R. 1981, Bull. Astron. Inst. Czechosl., 32, 93

Jones, M. H., \& Watson, M. G. 1992, MNRAS, 257, 633

Kim, Soon-Wook, Wheeler, J. C., \& Mineshige, S. 1992, ApJ, 384, 269

King, A. R., Ricketts, M. J., \& Warwick, R. S. 1979, MNRAS, 187, 77

Leach, R., Hessman, F. V., King, A. R., Stehle, R., \& Mattei, J. 1999, MNRAS, 305, 225

Mineshige, S., Tuchman, Y., \& Wheeler, J. C. 1990, ApJ, 359, 176

Lasota, J.-P., Kuulkers, E., \& Charles, P. 1999, MNRAS, 305, 473

Morales-Rueda, L., Still, M. D., \& Roche, P. 1996, MNRAS, 283, L58

Morales-Rueda, L., Still, M. D., \& Roche, P. 1999, MNRAS, 306, 753

Mumford, G. S. 1966, ApJ, 146, 411

Norton, A. J., Watson, M. G., \& King, A. R. 1988, MNRAS, 231,783

Percy, J. R., Fabro, V. A., \& Keith, D. W. 1985, J. AAVSO, 14, 1 
Pezzuto, S., Bianchini, A., \& Stagni, R. 1996, A\&A, 312, 865

Prialnik, D. 1986, ApJ, 310, 222

Richman, H. R., Applegate, J. H., \& Patterson, J. 1994, PASP, 106, 1075

Rosino, L., Bianchini, A., \& Rafanelli, P. 1982, A\&A, 108, 243

Schreiber, M. R., Gänsicke, B. T., \& Cannizzo, J. K. 2000, A\&A, 362, 268

Smak, J. 1984, Acta Astron., 34, 161

Sabbadin, F., \& Bianchini, A. 1983, A\&AS, 54, 393

Stellingwerf, R. F., 1978, ApJ, 224, 953

Szkody, P., Mattei, J. A., \& Mateo, M. 1985, PASP, 97, 264

Šimon, V. 2000a, A\&A, 354, 103

Šimon, V. 2000b, A\&A, 360, 627

Šimon, V. 2000c, A\&A, 364, 694
Vogt, N. 1980, A\&A, 88, 66

Vondrák, J. 1969, Bull. Astron. Inst. Czechosl., 20, 349

Vondrák, J. 1977, Bull. Astron. Inst. Czechosl., 28, 84

Walker, M. F. 1957, in Non-Stable Stars, ed. G. H. Herbig (Cambridge: Cambridge University Press), 46

Warner, B. 1995, Cataclysmic Variable Stars (Cambridge Univ. Press, Cambridge)

Watson, M. G., King, A. R., \& Osborne, J. 1985, MNRAS, 212,917

Wu, Ch.-Ch., Holm, A. V., Panek, R. J., et al. 1989, ApJ, 339, 443

Yi, I., Kim, S.-W., Vishniac, E. T., \& Wheeler, J. C. 1992, ApJ, 391, L25

Yi, I., \& Vishniac, E. T. 1994, ApJ, 435, 829 